PROCEEDINGS OF THE

AMERICAN MATHEMATICAL SOCIETY

Volume 127, Number 12, Pages 3593-3600

S 0002-9939(99)04938-2

Article electronically published on May 6, 1999

\title{
A RECONSTRUCTION FORMULA FOR BAND LIMITED FUNCTIONS IN $L_{2}\left(R^{d}\right)$
}

\author{
ISAAC PESENSON
}

(Communicated by Christopher D. Sogge)

\begin{abstract}
It is shown that a band limited function from $L_{2}\left(R^{d}\right)$ can be reconstructed from irregularly sampled values as a limit of spline functions. The assumption about the sampling sequence is that it should be dense enough.
\end{abstract}

It is known that band limited functions, i.e. functions whose Fourier transform have compact support, are uniquely determined and can be recovered from their values on specific discrete set of points $x_{n}$. The classical Whittaker-Shannon Theorem gives the sharp answer in the case of equally spaced points in $R$. The irregularly spaced sets of sampling points were considered by Paley and Wiener [6]. More recent results and extensive lists of references can be found in surveys of Benedetto [1] and Feichtinger and Gröchening [2].

We prove that a band limited function from $L_{2}\left(R^{d}\right)$ can be recovered from its values on specific irregularly distributed sampling points as the limit of $d$-dimensional polyharmonic spline functions. The assumption about the sampling sequence is that it should be dense enough. Our reconstruction formula is very stable due to the fact that fundamental splines have exponential decay. Moreover, we prove that convergence takes place not only in the space $L_{p}\left(R^{d}\right), 2 \leq p \leq \infty$, but also in the $C^{k}$ norm for any $k>0$. In particular, it shows the way to reconstruct all derivatives of a band limited function using samples of the function.

Splines as a tool for reconstruction in the one-dimensional case were used by Schoenberg [9] for equally spaced knots and recently by Lyubarskii and Madych [3] under the assumption that corresponding exponential functions form a Riesz basis in $L_{2}([-\pi, \pi])$.

Technically our paper is close to [5]. Our proofs rely explicitly on the observation that certain constants in inequalities from Lemmas 1 and 2 below have a very special form: they depend exponentially of the order of derivatives involved in inequalities. This allows us to control the convergence when the order of derivatives goes to infinity. This technique is very flexible and can be used to solve the reconstruction problem in new situations [7], [8]. A new application of this technique is also given at the end of this paper.

Let $X(\lambda)$ denote a countable set $\left\{x_{\gamma}\right\} \in R^{d}$ such that the rectangles $D\left(x_{\nu}, \lambda\right)$ of diameter $\leq \lambda$ each containing exactly one point $x_{\nu}$ form a disjoint cover of $R^{d}$.

Received by the editors August 23, 1997 and, in revised form, February 17, 1998.

1991 Mathematics Subject Classification. Primary 42A65; Secondary 42C15.

(C)1999 American Mathematical Society 
For the given $X(\lambda)$ the fundamental spline $L_{\nu}^{k}, k>d / 2$, is introduced as a function from $H^{k}\left(R^{d}\right), k>d / 2$, such that

1) $L_{\nu}^{k}\left(x_{\nu}\right)=1$ and is zero at all other points of the set $X(\lambda)$;

2 ) it minimizes the functional $u \rightarrow\left\|\Delta^{k / 2} u\right\|$ on $H^{k}\left(R^{d}\right), k>d / 2$, where $\Delta$ is the Laplace operator.

The set of band limited functions $B_{\sigma}\left(R^{d}\right)$ is the set of all $f \in L_{2}\left(R^{d}\right)$ such that the Fourier transform

$$
\hat{f}(\xi)=\int_{R^{d}} f(x) e^{-i x \xi} d x
$$

has support in the ball $B(0, \sigma)$ of radius $\sigma$ centered at 0 . The symbol $\|f\|$ means the $L_{2}\left(R^{d}\right)$-norm of $f$. Our main result is the following.

Theorem 1. There exists a constant $c_{0}=c_{0}(d)$ that depends only on dimension $d$ such that for any $\lambda>0$, every $X(\lambda)$ and all nonnegative integers $k$ and $r \geq[d / 2]+1$

$$
\left\|\Delta^{k}\left(f-\sum_{x_{\nu} \in X(\lambda)} f\left(x_{\nu}\right) L_{\nu}^{2^{l} r+k}\right)\right\|_{L_{p}\left(R^{d}\right)} \leq 2 \sigma^{2 k}\left(c_{0} \lambda \sigma\right)^{2^{l+1} r}\left(\sum_{x_{\nu} \in X(\lambda)}\left|f\left(x_{\nu}\right)\right|^{2}\right)^{1 / 2}
$$

for all $f \in B_{\sigma}\left(R^{d}\right), l=0,1,2, \ldots, 2 \leq p \leq \infty$.

Note that functions $L_{\nu}^{k}$ have very fast decay at infinity in the sense that for every $k$ there are $a>0, b>0$ such that $\left|L_{\nu}^{k}(x)\right| \leq a e^{-b\left|x_{\nu}-x\right|}$. This fact makes the above reconstruction formula very local.

Lemma 1. 1) If for some $f \in H^{2 s}\left(R^{d}\right), s>0, c>0$,

$$
\|f\| \leq c\left\|\Delta^{s} f\right\|
$$

then for the same $f, c$ and $s$ and all $\tau \geq 0, m=2^{l}, l=0,1,2, \ldots$,

$$
\left\|\Delta^{\tau} f\right\| \leq c^{m}\left\|\Delta^{m s+\tau} f\right\|
$$

as long as $f \in H^{2(m s+\tau)}\left(R^{d}\right)$.

2) If for some $A, c, s>0$ and $f \in H^{2 s}\left(R^{d}\right)$

$$
\|f\| \leq A+c\left\|\Delta^{s} f\right\|
$$

then for the same $A, c, s, f$

$$
\|f\| \leq 2 m A+8^{m-1} c^{m}\left\|\Delta^{m s} f\right\|
$$

for $m=2^{l}, l=0,1, \ldots$, as long as $f \in H^{2 m s}\left(R^{d}\right)$.

Proof. For the ball $B=B\left(0, c^{-1 / 2 s}\right)$ we have

$$
\int_{B}|\hat{f}|^{2} d \xi+\int_{R^{d} \backslash B}|\hat{f}|^{2} d \xi \leq c^{2}\left(\int_{B}|\xi|^{4 s}|\hat{f}|^{2} d \xi+\int_{R^{d} \backslash B}|\xi|^{4 s}|\hat{f}|^{2} d \xi\right) .
$$

Since $c^{2}|\xi|^{4 s}<1$ on $B\left(0, c^{-1 / 2 s}\right)$,

$$
0 \leq \int_{B}\left(|\hat{f}|^{2}-c^{2}|\xi|^{4 s}|\hat{f}|^{2}\right) d \xi \leq \int_{R^{d} \backslash B}\left(c^{2}|\xi|^{4 s}|\hat{f}|^{2}-|\hat{f}|^{2}\right) d \xi
$$


This inequality implies

$$
0 \leq \int_{B}\left(c^{2}|\xi|^{4 s}|\hat{f}|^{2}-c^{4}|\xi|^{8 s}|\hat{f}|^{2}\right) d \xi \leq \int_{R^{d} \backslash B}\left(c^{4}|\xi|^{8 s}|\hat{f}|^{2}-c^{2}|\xi|^{4 s}|\hat{f}|^{2}\right) d \xi
$$

or

$$
c^{2} \int_{R^{d}}|\xi|^{4 s}|\hat{f}|^{2} d \xi \leq c^{4} \int_{R^{d}}|\xi|^{8 s}|\hat{f}|^{2} d \xi
$$

and then

$$
\|f\| \leq c\left\|\Delta^{s} f\right\| \leq c^{2}\left\|\Delta^{2 s} f\right\| .
$$

Now, using induction one can show the inequality $\|f\| \leq c^{m}\left\|\Delta^{m s} f\right\|$ for any $m=2^{l}, l=0,1, \ldots$ Then again, because for any $t \geq 0, c^{2 t}|\xi|^{4 t s}<1$ on $B\left(0, c^{-1 / 2 s}\right)$ we have

$$
\begin{aligned}
0 & \leq \int_{B}\left(c^{2 t}|\xi|^{4 t s}|\hat{f}|^{2}-c^{2(m+t)}|\xi|^{4(m+t) s}|\hat{f}|^{2}\right) d \xi \\
& \leq \int_{R^{d} \backslash B}\left(c^{2(m+t)}|\xi|^{4(m+t) s}|\hat{f}|^{2}-c^{2 t}|\xi|^{4 t s}|\hat{f}|^{2}\right) d \xi
\end{aligned}
$$

this gives $\left\|\Delta^{t s} f\right\| \leq c^{m}\left\|\Delta^{(m+t) s} f\right\|$. Setting $t s=\tau \geq 0$ we obtain the desired inequality.

Next, for any $\varepsilon>0$

$$
\begin{aligned}
& \varepsilon\left\||\xi|^{s / 2} \hat{f}\right\| \leq\left\|\left(1-i \varepsilon|\xi|^{s / 2}\right) \hat{f}\right\|+\|\hat{f}\| \\
& \quad \leq\left\|\left(1+\varepsilon^{2}|\xi|^{s}\right) \hat{f}\right\|+\|\hat{f}\| \leq \varepsilon^{2}\left\||\xi|^{s} \hat{f}\right\|+2\|\hat{f}\|
\end{aligned}
$$

or

$$
\left\|\Delta^{s} f\right\| \leq \varepsilon\left\|\Delta^{2 s} f\right\|+\frac{2}{\varepsilon}\|f\|
$$

The assumption of the lemma corresponds to the case $m=1$. If it is true for $m$, then

$$
\|f\| \leq m A+8^{m-1} c^{m}\left(\varepsilon\left\|\Delta^{2 m s} f\right\|+\frac{2}{\varepsilon}\|f\|\right) .
$$

Choosing $\varepsilon=2^{3 m-1} c^{m}$ we obtain

$$
\|f\| \leq 2 m A+8^{2 m-1} c^{2 m}\left\|\Delta^{2 m s} f\right\| .
$$

The lemma is proved.

Corollary 1. If a function satisfies an inequality $\|f\| \leq c\left\|\Delta^{s} f\right\|$ for some $c>0$, $s>0$, then the support of its Fourier transform $\hat{f}$ has nonempty intersection with $R^{d} \backslash B\left(0, c^{-1 / 2 s}\right)$.

As an application of Lemma 1 we can extend the Wirtingen inequality

$$
\|f\| \leq \frac{b-a}{\pi}\|d f / d t\|, \quad f(a)=f(b)=0, f \in H^{1}(R),
$$

to

$$
\left\|\left(\frac{d}{d t}\right)^{k} f\right\| \leq\left(\frac{\lambda}{\pi}\right)^{r-k}\left\|\left(\frac{d}{d t}\right)^{r} f\right\|, \quad k, r=0,1,2, \ldots, f \in H^{r}(R),
$$

where $f$ has zeros at points $x_{\gamma}$ and $\max _{\gamma}\left|x_{\gamma}-x_{\gamma+1}\right|=\lambda$. 
In fact the operator $\frac{d}{d t}$ is not a power of $\frac{d^{2}}{d t^{2}}$ but the above proof goes through for $\frac{d}{d t}$ and its natural powers. So, for $s=m=1, \tau=k$,

$$
\left\|\left(\frac{d}{d t}\right)^{k} f\right\| \leq \frac{\lambda}{\pi}\left\|\left(\frac{d}{d t}\right)^{k+1} f\right\| ;
$$

this implies the desired inequality.

The symbol $U^{s}(X(\lambda)), s>d / 2$, denotes the set of all functions $f$ from $H^{s}\left(R^{d}\right)$, $s>d / 2$, such that $\left.f\right|_{X(\lambda)}=0$.

Lemma 2. There exists a constant $c_{0}=c_{0}(d)$ such that for every $\lambda>0$ and every $X(\lambda)$

$$
\left\|\Delta^{\tau} f\right\| \leq\left(c_{0} \lambda\right)^{2 m s}\left\|\Delta^{m s+\tau} f\right\|
$$

for all $s \geq[d / 2]+1, \tau \geq 0, m=2^{l}, l=0,1,2, \ldots, f \in U^{2(m s+\tau)}(X(\lambda))$.

Proof. If $f \in H^{k}\left(R^{d}\right), k>d / 2$, then

$$
\|f\|_{L^{2}\left(D\left(x_{\gamma}, \lambda\right)\right)} \leq C\left(\lambda^{d / 2}\left|f\left(x_{\gamma}\right)\right|+\sum_{j=1}^{k} \lambda^{j} \sum_{|\alpha|=j}\left\|\partial^{\alpha} f\right\|_{L^{2}\left(D\left(x_{\gamma}, \lambda\right)\right)}\right)
$$

where $C$ depends only on $k, d$. Indeed, the Taylor formula gives that on $D\left(x_{\gamma}, \lambda\right)$ the function $f(x)-f\left(x_{\gamma}\right)$ is a linear combination of $\partial^{\alpha} f(x)\left(x-x_{\gamma}\right)^{\alpha}$ with $1 \leq|\alpha|<k$, and $\int_{0}^{\rho} t^{k-1} \partial^{\alpha} f\left(x_{\gamma}+t \theta\right) \theta^{\alpha} d t$ with $|\alpha|=k$, where $\alpha=\left(\alpha_{1}, \ldots, \alpha_{d}\right),\left(x-x_{\gamma}\right)^{\alpha}=$ $\left(x_{1}-x_{1, \gamma}\right)^{\alpha_{1}} \cdots\left(x_{d}-x_{d, \gamma}\right)^{\alpha_{d}}, \theta^{\alpha}=\theta_{1}^{\alpha_{1}} \cdots \theta_{d}^{\alpha_{d}}, \theta=\left(x-x_{\gamma}\right) / \rho, \rho=\left|x-x_{\gamma}\right|$ and $\partial^{\alpha}$ is a mixed partial derivative. It is evident that the first group of terms is dominated by

$$
C \sum_{j=1}^{k-1} \lambda^{j} \sum_{1 \leq|\alpha| \leq j}\left\|\partial^{\alpha} f\right\|_{L^{2}\left(D\left(x_{\gamma}, \lambda\right)\right)}
$$

for some $C=C(k, d) \geq 0$. Next, using the Schwartz inequality we obtain for $k>d / 2$

$$
\left|\int_{0}^{\rho} \partial^{\alpha} f\left(x_{\gamma}+t \theta\right) \theta^{\alpha} d t\right|^{2} \leq C \rho^{2 k-d} \int_{0}^{\rho}\left|\partial^{\alpha} f\left(x_{\gamma}+t \theta\right)\right|^{2} t^{d-1} d t, \quad C=C(k, d) .
$$

We integrate both sides of this inequality in the spherical coordinate system $(\rho, \theta)$. Changing the order of integration in $t$ and $\rho$ we obtain that the $L_{2}\left(D\left(x_{\gamma}, \lambda\right)\right)$ norm of the term

$$
\int_{0}^{\rho} t^{k-1} \partial^{\alpha} f\left(x_{\gamma}+t \theta\right) \theta^{\alpha} d t
$$

is dominated by $C \lambda^{k} \sum_{|\alpha|=k}\left\|\partial^{\alpha} f\right\|_{L_{2}\left(D\left(x_{\gamma} \lambda\right)\right)}$, where $C=C(k, d) \geq 0$. Summation over all rectangles in the cover gives

$$
\|f\| \leq C(d, k)\left(\lambda^{d / 2}\left(\sum\left|f\left(x_{\gamma}\right)\right|^{2}\right)^{1 / 2}+\sum_{j=1}^{k} \lambda^{j} \sum_{|\alpha|=j}\left\|\partial^{\alpha} f\right\|\right) .
$$

Repeated applications of the interpolating inequality

$$
\|f\|_{H^{j}\left(R^{d}\right)} \leq C(d, k)\left(\varepsilon^{-j}\|f\|+\varepsilon^{k-j}\|f\|_{H^{k}\left(R^{d}\right)}\right)
$$


with careful choice of the corresponding $\varepsilon$, lead to the inequality

$$
\|f\| \leq C(d, k)\left(\lambda^{d / 2}\left(\sum_{\gamma}\left|f\left(x_{\gamma}\right)\right|^{2}\right)^{1 / 2}+\lambda^{k} \sum_{|\alpha|=k}\left\|\partial^{\alpha} f\right\|\right) .
$$

Using the fact that

$$
\|f\|+\sum_{|\alpha| \leq k}\left\|\partial^{\alpha} f\right\| \leq C(d, k)\left(\|f\|+\left\|\Delta^{k / 2} f\right\|\right)
$$

we obtain that if $\lambda$ is smaller than some $\lambda_{0}$ that depends on dimension $d$ and $k$, then

$$
\|f\| \leq C(d, k)\left(\lambda^{d / 2}\left(\sum_{\gamma}\left|f\left(x_{\gamma}\right)\right|^{2}\right)^{1 / 2}+\lambda^{k}\left\|\Delta^{k / 2} f\right\|\right),
$$

for $\lambda<\lambda_{0}(d, k)$.

But now using rescaling arguments one can show that this inequality takes place for any $\lambda>0$. Applying the second part of Lemma 1 to the last inequality with $k_{0}=[d / 2]+1$ we obtain

$$
\|f\| \leq 2 m c_{0}\left(\sum_{\gamma}\left|f\left(x_{\gamma}\right)\right|^{2}\right)^{1 / 2}+\left(c_{0} \lambda\right)^{m k_{0}}\left\|\Delta^{m k_{0} / 2} f\right\|, \quad \lambda>0,
$$

for any $m=2^{l}, l-0,1, \ldots$, where $c_{0}$ depends only on $d$. In particular, for $f \in$ $U^{2 m k}(X(\lambda))$ we have

$$
\|f\| \leq\left(c_{0} \lambda\right)^{m k_{0}}\left\|\Delta^{m k_{0} / 2} f\right\|, \quad k_{0}=[d / 2]+1, \lambda>0,
$$

where $c_{0}$ depends only on dimension $d$. We are going to extend this inequality to all $s>d / 2$. Note that the norm

$$
\left\|\Delta^{k / 2} f\right\|+\left(\sum_{\gamma}\left|f\left(x_{\gamma}\right)\right|^{2}\right)^{1 / 2}
$$

is equivalent to the Sobolev norm. Indeed, in one direction it is already proved. In the opposite direction, using Sobolev's embedding theorem we obtain for any natural $k>d / 2$

$$
\left(\sum_{\gamma}\left|f\left(x_{\gamma}\right)\right|^{2}\right)^{1 / 2} \leq C(d, k)\left(\sum_{\gamma}\left\|f \phi_{\gamma}\right\|_{H^{k}\left(R^{d}\right)}^{2}\right)^{1 / 2} \leq C(d, k)\|f\|_{H^{k}\left(R^{d}\right)} .
$$

In particular, on $U^{k}(X(\lambda)), k>d / 2$, the norm $\left\|\Delta^{k / 2} f\right\|$ is equivalent to the Sobolev norm. Thus, our previous estimates can be reformulated in the following way: the identity operator is a continuous map from $U^{m k_{0}}(X(\lambda)), k_{0}=[d / 2]+1, m=2^{l}, l=$ $0,1, \ldots$, with $H^{m k_{0}}\left(R^{d}\right)$-norm into $U^{m k_{0}}(X(\lambda))$ with $L_{2}\left(R^{d}\right)$-norm and its norm is bounded by $\left(c_{0} \lambda\right)^{m k_{0}}$. The interpolation arguments show that then

$$
\|f\| \leq\left(c_{0} \lambda\right)^{2 s}\left\|\Delta^{s} f\right\|, \quad \lambda>0, s \geq[d / 2]+1
$$

for all $f \in U^{2 s}(X(\lambda))$. Using Lemma 1 again we obtain

$$
\left\|\Delta^{\tau} f\right\| \leq\left(c_{0} \lambda\right)^{2 m s}\left\|\Delta^{m s+\tau} f\right\|, \quad f \in U^{2(m s+\tau)}(X(\lambda)), \lambda>0,
$$

where $s \geq[d / 2]+1, \tau \geq 0, m=2^{l}, l=0,1, \ldots$. 
Many consequences can be derived from this inequality. For instance, if $\tau=$ $s, m=1$, then

$$
\left\|\Delta^{s} f\right\| \leq\left(c_{0} \lambda\right)^{2 s}\left\|\Delta^{2 s} f\right\|, \quad s \geq[d / 2]+1, f \in U^{4 s}(X(\lambda)), \quad \lambda>0 .
$$

Iterations of this inequality give

$$
\left\|\Delta^{s} f\right\| \leq\left(c_{0} \lambda\right)^{2(m-1) s}\left\|\Delta^{m s} f\right\|, \quad s \geq[d / 2]+1, m=2^{l}, l=0,1, \ldots,
$$

$f \in U^{4 s}(X(\lambda)), \lambda>0$. Lemma 2 is proved.

Our next goal is to introduce splines using the variational method. Given a $X(\lambda)$ and a sequence $\left\{s_{\gamma}\right\} \in l_{2}$ we will be interested to find a function $h_{k} \in H^{k}\left(R^{d}\right)$, $k>d / 2$ :

a) $h_{k}\left(x_{\gamma}\right)=s_{\gamma}$, for $x_{\gamma} \in X(\lambda)$

b) $h_{k}$ minimizes functional $u \rightarrow\left\|\Delta^{k / 2} u\right\|$.

If $f$ is any function from $H^{k}\left(R^{d}\right)$ such that $f\left(x_{\gamma}\right)=s_{\gamma}$ and $P f$ denotes the orthogonal projection of this function $f$ in the Hilbert space $H^{k}\left(R^{d}\right)$ with inner product

$$
\langle f, g\rangle=\sum_{x_{\gamma}} f\left(x_{\gamma}\right) g\left(x_{\gamma}\right)+\left\langle\Delta^{k / 2} f, \Delta^{k / 2} g\right\rangle
$$

on the subspace $U^{k}(X(\lambda))$ with $H^{k}\left(R^{d}\right)$-norm, then the function $g=f-P f$ will be a unique solution of the above minimization problem.

It is known [4] that a function $h_{k}, h_{k}\left(x_{\nu}\right)=s_{\nu}$, is a solution to the minimization problem stated above if and only if

$$
\Delta^{k} h_{k}=\sum c_{\nu} \delta_{\nu}, \quad\left\{c_{\nu}\right\} \in l_{2}
$$

where $\delta_{\nu}$ is the Dirac measure at $x_{\nu}$. Every such function $h_{k}$ is of the form $\sum s_{\nu} L_{\nu}^{k}$ where $L_{\nu}^{k}$ is the fundamental spline: $L_{\nu}^{k}\left(x_{\nu}\right)=1$ and zero for all other $x_{\gamma}$.

Recall that a function $f$ belongs to $B_{\sigma}\left(R^{d}\right)$ if and only if it satisfies the Bernstein inequality $\left\|\Delta^{s / 2} f\right\| \leq \sigma^{s}\|f\|, s \geq 0$.

Next, for any $f \in L_{2}\left(R^{d}\right)$ using Lemma 2, the minimization property and Bernstein inequality we obtain for any nonnegative integer $k$ and $r \geq[d / 2]+1$

$$
\begin{aligned}
& \left\|\Delta^{k}\left(f-\sum_{x_{\nu} \in X(\lambda)} f\left(x_{\nu}\right) L_{\nu}^{2^{l} r+k}\right)\right\| \\
& \quad \leq\left(c_{0} \lambda\right)^{\left(2^{l+1} r\right)}\left\|\Delta^{2^{l} r+k}\left(f-\sum_{x_{\nu} \in X(\lambda)} f\left(x_{\nu}\right) L_{\nu}^{2^{l} r+k}\right)\right\| \\
& \quad \leq 2\left(c_{0} \lambda\right)^{\left(2^{l+1} r\right)}\left\|\Delta^{2^{l} r+k} f\right\| \leq 2 \sigma^{2 k}\left(c_{0} \lambda \sigma\right)^{2^{l+1} r}\|f\|,
\end{aligned}
$$

that goes to zero if $l \rightarrow \infty$ and $c_{0} \lambda \sigma<1$. Note that $\|f\|$ can be replaced by $\left(\Sigma_{\gamma}\left|f\left(x_{\gamma}\right)\right|^{2}\right)^{1 / 2}$. To complete the proof of Theorem 1 it is enough to use the Sobolev embedding theorem and interpolation theory. Theorem 1 is proved. 
As a consequence of the proof we can formulate the following approximation result.

Corollary 2. If $f \in H^{2^{l+1} r+2 k}\left(R^{d}\right)$, then

$$
\begin{aligned}
& \left\|\Delta^{k}\left(f-\sum_{x_{\nu} \in X(\lambda)} f\left(x_{\nu}\right) L_{\nu}^{2^{l} r+k}\right)\right\|_{L_{p}\left(R^{d}\right)} \\
& \quad \leq 2\left(c_{0} \lambda\right)^{\left(2^{l+1} r\right)}\left\|\Delta^{2^{l} r+k} f\right\|, \quad l=0,1, \ldots, r \geq[d / 2]+1,
\end{aligned}
$$

where $2 \leq p \leq \infty$. Using interpolation theory one can extend this result to Besov spaces.

Note that all our proofs can be performed in a more general setting. Let $D$ be a second order self-adjoint, positive definite operator in $L_{2}\left(R^{d}\right)$. We assume that the coefficients of $D$ and all their derivatives are bounded. Then the domain of $D^{k}$ is $H^{2 k}\left(R^{d}\right)$ and the Sobolev norm is equivalent to $\|f\|+\left\|D^{k} f\right\|$. All previous estimates remain true for $D^{k}$ instead of $\Delta^{k}$. The only difference is that such an operator is not necessarily homogeneous and we will not be able to use rescaling arguments. As a result all inequalities will be true only for small values of $\lambda$.

We consider the following minimization problem: given a discrete set of points $X(\lambda)$ and a sequence $f\left(x_{\gamma}\right)=\left\{s_{\gamma}\right\} \in l_{2}, f \in H^{k}\left(R^{d}\right), k>d / 2$, find a function $h_{k}(f) \in H^{k}\left(R^{d}\right), k>d / 2$, such that

a) $h_{k}(f)\left(x_{\gamma}\right)=s_{\gamma}$ for $x_{\gamma} \in X(\lambda)$, and

b) the function $h_{k}(f)$ minimizes the functional $u \rightarrow\left\|D^{k / 2} u\right\|$.

The analog of band limited functions is introduced by

$$
B_{\sigma}(D)=\left\{f_{2}:\left\|D^{k / 2} f\right\| \leq \sigma^{k}\|f\|\right\} .
$$

As the spectral theorem shows, the union of $B_{\sigma}\left(R^{d}\right)$ for all $s \geq 0$ is dense in $L_{2}\left(R^{d}\right)$.

Theorem 2. There exist $c(D), \lambda_{0}(D)$ such that if $\lambda<\lambda_{0}(D)$, then for any $f \in$ $B_{\sigma}\left(R^{d}\right)$

$$
\left\|D^{k}\left(f-h_{2^{l} r+k}(f)\right)\right\|_{L_{p}\left(R^{d}\right)} \leq 2 \sigma^{2 k}(c(D) \lambda \sigma)^{2^{l+1} r}\|f\|, \quad l=0,1, \ldots,
$$

for any nonnegative integer $k$ and $r \geq[d / 2]+1$ and $2 \leq p \leq \infty$.

\section{REFERENCES}

1. J. J. Benedetto, Irregular sampling and frames, in Wavelets: A Tutorial in Theory and Applications (C. K. Chui, Ed.), 445-507. Academic Press, Boston, 1992. MR 93c:42030

2. H. G. Feichtinger and K. Gröchening, Theory and practice of irregular sampling, in Wavelets (J. J. Benedetto and M. W. Frazier, Eds.) pp. 305-363, CRC Press, Boca Raton, FL, 1994. MR 94i:94008

3. Yu. Lyubarskii and W. R. Madych, The recovery of irregularly sampled band limited functions via tempered splines, J. Funct. Anal. 125 (1995), 201-222. MR 96d:41013

4. W. R. Madych and S. A. Nelson, Polyharmonic cardinal splines, J. Approximation Theory 60 (1990), 141-156. MR 90j:41022

5. W. R. Madych and E. H. Potter, An estimate for multivariate interpolation, J. Approximation Theory 43 (1985), 132-139. MR 86g:65022

6. R. Paley and N. Wiener, Fourier transforms in the complex domain, in Amer. Math. Soc. Colloq. Publ., Vol. 19, Amer. Math. Soc., Providence, 1934. MR 98a:01023

7. I. Pesenson, Sampling of Paley-Wiener functions on stratified groups, J. of Fourier Analysis and Applications 4 (3) (1998), 271-281. CMP 99:03 
8. I. Pesenson, Reconstruction of Paley-Wiener functions on the Heisenberg group, Amer. Math. Soc. Transl. (2), vol. 184, 1998.

9. I, Schoenberg, Cardinal spline interpolation, CBMS, Vol. 12, SIAM, Philadelphia, 1973. MR 54:8095

Department of Mathematics 038-16, Temple University, Philadelphia, Pennsylvania 19122

E-mail address: pesenson@math.temple.edu 